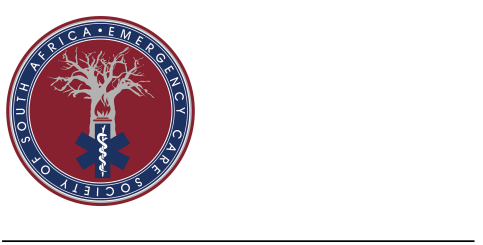

ARTICLE INFORMATION

Received: 15 January 2019

Revision received: 26 February 2019

Accepted: 7 March 2019

Keywords:

Emergency Medical Services

risk management

clinical protocols

clinical decision-making

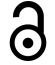

This open access article is distributed under Creative Commons licence CCBY-NC 4.0

\section{On-scene discharge by Emergency Care Practitioners - a viable option for South Africa?}

\author{
Lara Nicole Goldstein*, Siyabonga Sibanyoni, Craig Vincent-Lambert \\ Department of Emergency Medical Care, Faculty of Health Sciences, \\ University of Johannesburg
}

"Corresponding author:drg666@gmail.com, Postnet Suite 429, Private Bag X1510, Glenvista, 2058

\begin{abstract}
Background: In South Africa, the national public health sector provides healthcare to the majority of the population, yet many hospitals are in a state of crisis. On-scene discharge or patient non-conveyance to hospital by Emergency Care Practitioners (ECP) may serve as a means for patients to access healthcare services while alleviating the burden on the hospitals. The aim of this study was to determine the number of patients being transported by ambulance to a public sector Emergency Department (ED) who could have potentially been managed and discharged on-scene by a South African pre-hospital ECP. Methods: This was a prospective, descriptive and quantitative study of patients brought to the ED by ambulance over a 72-hour weekend period. The medical care that was provided in the ED was compared to the existing ECP scope of practice. The patients' disposition was also used as a surrogate to determine whether transportation to hospital was required.

Results: A total of 118 patients were transported to the ED by ambulance, and 85 of these patients consented to participate in the study. Overall, $62.4 \%$ of these patients were ultimately discharged from the hospital (60.4\% of discharged patients being trauma-related). Most of the treatment modalities prescribed for the discharged patients were within the ECP scope of practice.

Conclusion: Pre-hospital on-scene discharge by ECPs may be a mechanism to alleviate hospital overcrowding in a failing public healthcare system. However, if an ambulance service condones the concept of on-scene discharge, they need to implement monitoring strategies to assess the subsequent outcomes for those patients discharged at the scene.
\end{abstract}

Goldstein LN, Sibanyoni S, Vincent-Lambert C. On-scene discharge by Emergency Care Practitioners - a viable option for South Africa? South African Journal of Pre-hospital Emergency Care. 2020; 1(1):3-8. doi:10.24213/1-1-3330

\section{BACKGROUND}

In South Africa, the national public health sector provides healthcare to the majority of the population, ${ }^{1}$ yet many public sector hospitals are in a state of crisis - their infrastructure is often dysfunctional and run down. This includes the referral system from primary healthcare facilities to tertiary hospitals. South Africa also has the compounded problem of low nurse- and doctor-to-population ratios. ${ }^{2,3}$ This means that many areas of the country not only have limited access to in-hospital medical care, but also that the current hospital system is overwhelmed. ${ }^{2}$

These system limitations extend into the Emergency Department (ED). Overcrowding, access 
and exit block have been linked to a range of adverse outcomes for both patients and staff. ${ }^{4}$ The excess patient mortality and morbidity occur irrespective of whether the patients are admitted or discharged. ${ }^{4}$ Various ways of alleviating these problems are continually being sought.

A reduction in the number of patients arriving to the ED by ambulance has been suggested as a possible solution to ease the overloaded system. Patients can be assessed on-scene and a decision can be made whether transport to hospital is truly required; ${ }^{5}$ paramedics and emergency physicians agree that a significant percentage of patients do not, in fact, require ambulance transportation to the hospital. ${ }^{6}$

On-scene discharge or patient non-conveyance by Emergency Care Practitioners (ECP) in South Africa may serve to fulfil the criteria set out by the Patient's Rights' Charter for patients "to utilise the health care system properly" while still being able to "access health care services". " That said, patient safety is paramount when considering emergency medical service systems for onscene discharge. Prior studies looking into utilising paramedics and nurses to perform on-scene discharge have reported differing levels of success. ${ }^{8-10}$ Moreover, concerns around patient safety are reflected in the Health Professions Council of South Africa's Clinical Practice Guidelines for emergency care providers wherein it is indicated that, "stringent continuous quality improvement measures must be in place if a[n Emergency Medical] service implements on-scene discharge practices". ${ }^{11}$

A professional bachelor's degree, such as that completed by an ECP, demands high intellectual independence and development of research capacity in the methodology and techniques of that discipline. ${ }^{12}$ South African ECPs are registered as independent pre-hospital practitioners with the Health Professions Council of South Africa. To register and practice as an ECP, one must have completed a four-year full-time professional bachelor's degree in emergency medical care through a university or completed a National Diploma followed by a Bachelor of Technology degree. ${ }^{13}$ A major component of the bachelor's degree programmes focuses on patient assessment, diagnostics and clinical decision-making. Such skills and abilities arguably place the ECP in a position to conduct onscene discharge and make direct referrals within the healthcare system. However, in many regions, the scope of practice and systems required to support on-scene discharge may not be well-defined or developed. Consequently, current practices are such that the majority of patients are transported to the ED of receiving facilities, regardless of the nature of their complaint or their level of acuity. ${ }^{14}$

The aim of this study was to determine the number of patients being transported by ambulance to a public sector ED who could have been potentially managed and discharged on-scene by a South African pre-hospital ECP.

\section{DESIGN AND METHODS}

This was a prospective, descriptive and quantitative study which took place at a tertiary academic public hospital in a metropolitan area of Johannesburg, South Africa. The hospital serves a population of approximately 1 million people and sees 65 000 patients annually. ${ }^{15}$

Ethical approval for this study was obtained from the University of Johannesburg's Faculty of Health Sciences, Research Ethics Committee [REC-01-992016] as well as the head of the ED and Chief Executive Officer of the hospital. Informed consent was obtained from all enrolled patients.

Data were collected over a period of 72 hours Friday, 21 October 2016 until Monday, 24 October 2016. The selection of this time interval was purposeful in that it would include the weekend timeframe when the outpatient departments are closed. The following data were gathered:

1. The total number of cases brought to the ED by ambulance.

2. The total number of patients arriving by ambulance who were discharged from the ED; i.e. how many were sent home after assessment and/or treatment by the doctor.

3. The medical care that was provided in the ED to patients brought by ambulance; i.e. medical interventions, investigations and related care.

4. The above data were compared to the 2018 ECP scope of practice. ${ }^{11}$

\section{Data Analysis}

The raw data were captured on an Excel® spreadsheet for collation. The data were then analysed using simple descriptive statistics.

\section{RESULTS}

There were 450 patients seen in the ED during the specified 72-hour study period. A total of 118 patients were transported to the ED by ambulance. Consent was obtained from 85 of those patients to participate in the study. The patients' demographics are presented in Table 1 (sex and age were not collected). 
Table 1: Patient Demographics

\begin{tabular}{|c|c|c|c|}
\hline Triage Category* & Admitted n (\%) & Discharged n (\%) & Total \\
\hline Red & $0(0)$ & $1(100)$ & $1(1.2)$ \\
\hline Orange & $16(66.7)$ & $8(33.3)$ & $24(28.2)$ \\
\hline Yellow & $14(36.8)$ & $24(63.2)$ & $38(44.7)$ \\
\hline Green & $2(9.1)$ & $20(90.9)$ & $22(25.9)$ \\
\hline Type of Pathology & Admitted n (\%) & Discharged n (\%) & Total \\
\hline Trauma & $10(31.3)$ & $32(60.4)$ & $42(49.4)$ \\
\hline Non-trauma & $22(68.8)$ & $21(39.6)$ & $43(50.6)$ \\
\hline Total & $32 / 85(37.6)$ & $53 / 85(62.4)$ & \\
\hline
\end{tabular}

*Triage category was according to the South African Triage Scale: Red - resuscitation; Orange - those to be seen within 10 minutes of ED arrival; Yellow - those to be seen within 1 hour of ED arrival; and Green - non-urgent.

Special Investigations Performed in Hospital on Medical and Trauma Patients

The most frequently undertaken special investigations for both the trauma and medical (non- trauma) patients are shown in Figure 1. Thirtythree $(62.3 \%)$ of the discharged patients received at least one special investigation.

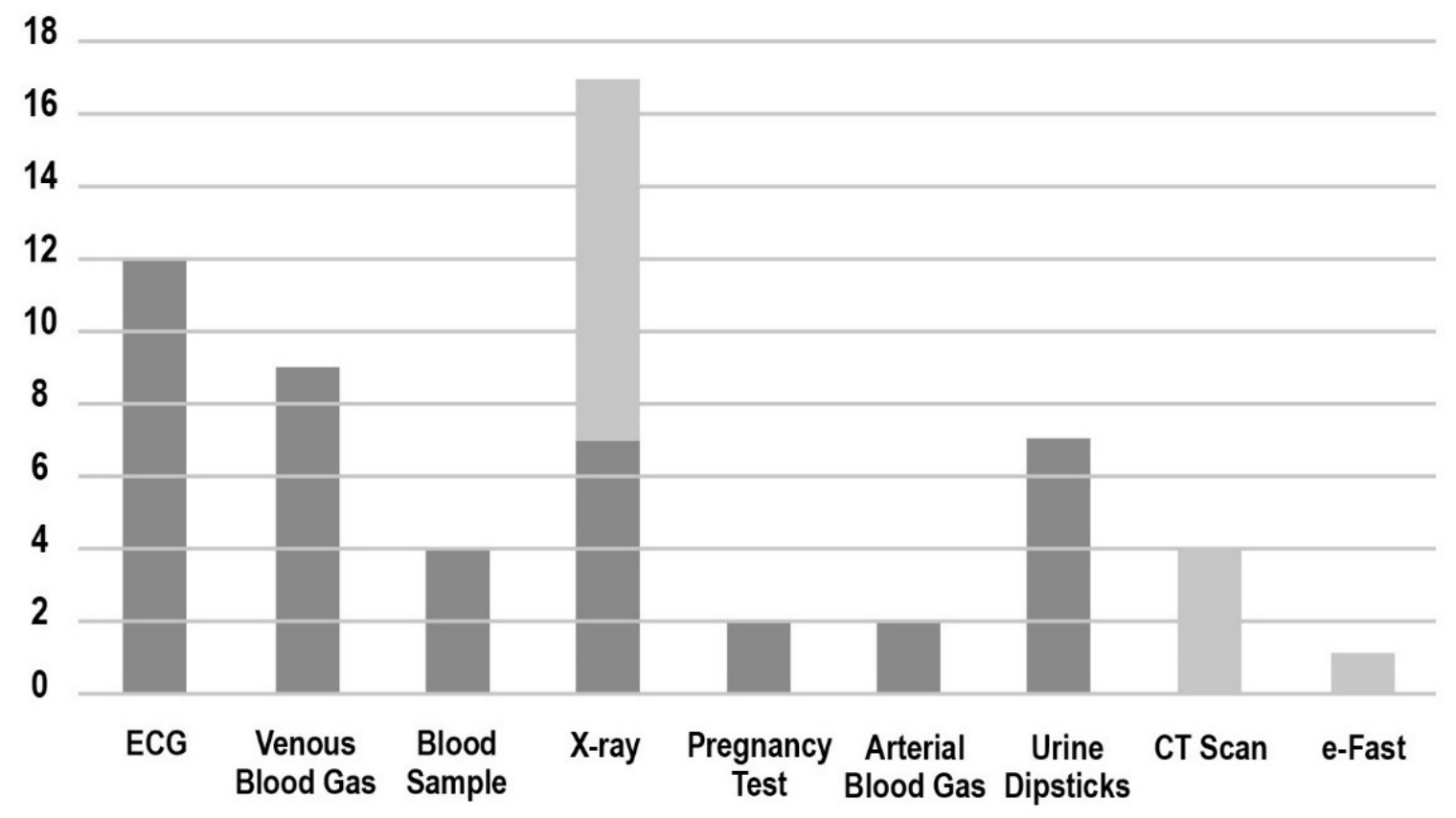

Medical

Trauma

Figure 1: Hospital Special Investigations Conducted in Trauma and Medical Patients

$\mathrm{CT}=$ scan computed tomography scan, ECG=electrocardiogram, e-FAST=extended focused assessment with sonar in trauma.

\section{Presenting Symptom Systems and Mechanism of Injury of Discharged Patients}

The presenting symptom systems of the medical patients and mechanisms of injury for the trauma patients who were discharged from the ED are presented in Table 2.

\section{Hospital Treatment Provided for Discharged Patients Compared to ECP Scope of Practice}

Table 3 tabulates the treatments administered in the ED compared with the ECP scope of practice. 
Table 2: Presenting Symptom Systems and Trauma Mechanisms of Injury: Discharged Patients

\begin{tabular}{ll}
\hline Medical Presenting Symptom Systems & $\mathbf{n}(\mathbf{\%})$ \\
\hline Gastrointestinal & $8(36.4)$ \\
Neurological & $4(18.2)$ \\
Gynaecological & $2(9.1)$ \\
Dermatological & $2(9.1)$ \\
Respiratory & $1(4.5)$ \\
Cardiovascular & $1(4.5)$ \\
Metabolic & $1(4.5)$ \\
Urological & $1(4.5)$ \\
Endocrine & $1(4.5)$ \\
Musculoskeletal & $1(4.5)$ \\
\hline Mechanism of Injury & $\mathbf{n}(\%)$ \\
\hline Motor Vehicle Accident & $14(46.7)$ \\
Common Assault & $11(36.6)$ \\
Penetrating Trauma & $3(10.0)$ \\
Pedestrian Vehicle Accident & $1(3.3)$ \\
Other Causes & $1(3.3)$ \\
\hline
\end{tabular}

Table 3: Hospital Treatment Provided for Discharged Patients vs ECP Scope of Practice

\begin{tabular}{|c|c|c|}
\hline Hospital Treatment & n (\%) & ECP Scope \\
\hline Analgesia & $24(46.2)$ & Yes \\
\hline Intravenous Therapy & $23(44.2)$ & Yes \\
\hline Suturing & $10(19.2)$ & Yes \\
\hline Wound Dressing & $1(1.9)$ & Yes \\
\hline Crutches $^{a}$ & $1(1.9)$ & Yes \\
\hline Thiamine & $1(1.9)$ & Yes \\
\hline Aspirin & $1(1.9)$ & Yes \\
\hline Anti-tetanus Toxoid & $5(9.6)$ & No \\
\hline Intravenous Antacid & $4(7.7)$ & No \\
\hline Intravenous anti-epileptics (e.g. Valproic acid) & $2(3.8)$ & No \\
\hline Topical Steroids & $1(1.9)$ & No \\
\hline
\end{tabular}

\section{DISCUSSION}

An overburdened and dysfunctional public health system needs all the help it can get when it comes to alleviating the pressure on the system itself. Decreasing the number of unnecessary ambulance cases presenting to the hospital is one such potential intervention.

\section{Mechanisms to Determine the Appropriateness of On-scene Discharge}

The determination of which patients should and should not be transported to the ED is a perpetual dilemma in the pre-hospital setting. ${ }^{9}$ Paramedics' ability to determine which patients can safely be treated in the community is, unfortunately, commonly inaccurate. ${ }^{4,8,9}$ This can lead to increased patient morbidity and mortality.
Potential confounders with regards to the determination of appropriateness for on-scene discharge, however, may stem from the comparator. Different outcomes in terms of the appropriateness of the paramedic decision will depend on whether the decision was compared to the patient's actual disposition from the ED (i.e. discharge and admission, as well as the occurrence of subsequent events such as ambulance re-request), whether the patient presented for a second ED visit, admission and/or death. ${ }^{16}$ All prior studies point to the dangers of paramedic under-triage, resulting in patients being left in the community rather than being brought to hospital. $5,6,8,9,16$ 


\section{Triage as a Mechanism to Determine the Appro- priateness of On-scene Discharge}

The South African Triage Scale is an in-hospital ED triage system. Patients who are triaged green are so-called 'non-urgent' patients, and they can be seen at a primary healthcare clinic. ${ }^{17}$ These patients may also potentially be discharged on-scene by an ECP due to their low acuity. Almost onequarter of patients who were brought to the hospital could therefore potentially not have been conveyed to the hospital. This is reinforced by the fact that over $90 \%$ of these patients were subsequently discharged home. The South African Triage Scale could be applied to the patient in the pre-hospital setting along with the ECP's clinical judgement and scope of practice in order to decrease patient conveyance to hospital. In our study, the ED nurses performed the triage. There could potentially have been more patients triaged green and therefore amenable to on-scene discharge this group of patients is commonly over-triaged. ${ }^{17}$

\section{Type of Pathology as a Mechanism to Deter- mine the Appropriateness of On-scene Dis- charge}

The majority $(70 \%)$ of patients presenting to this ED are usually non-traumatic in aetiology. As our data collection specifically took place over a weekend, it was unsurprising that there was a higher rate of trauma presentations. Trauma patients had fewer special investigations performed and were more likely to get discharged from the ED. This is similar to other studies where trauma patients had the lowest probability of hospital admission. 8,9 The trauma group of patients also had fewer inhospital procedures performed, making their onscene discharge a potential option. There were also more possible procedures that could be performed by ECP on the trauma patients. The provision of anti-tetanus toxoid into the ECP scope of practice would also make this more feasible.

\section{Presenting Symptoms and Mechanism of Injury to Determine the Appropriateness of On-scene Discharge}

The small sub-group of discharged medical patients' presenting symptoms and trauma patients' mechanisms of injury make meaningful suggestions for on-scene discharge impossible based on this data.

\section{Consequences of On-scene Discharge}

Delays in the initiation of treatment secondary to an incorrect on-scene discharge decision may lead to dire consequences for patients. The risks of the patient's condition deteriorating need to be weighed up against the potential burden of an unnecessary ambulance conveyance to hospital. ${ }^{6,9}$ Despite the current ECP clinical practice guidelines stating that continuous quality improvement measures should be in place in order to implement onscene discharge, there are currently no published data on the discharge practices of the feasibility thereof in Emergency Medical Services in South Africa. ${ }^{11}$

If an ambulance service condones the concept of on-scene discharge, they need to implement monitoring strategies to assess the subsequent outcomes for those patients discharged at the scene. The outcomes of interest include ambulance re-request, ED presentation (via alternate transportation), hospital admission, and death within 24 hours of the initial callout. $^{16}$

\section{LIMITATIONS}

This was not a prospective study that followed the patients from the scene to the hospital. The patients' pre-hospital condition was not utilised as a means of determining suitability for hospital non-conveyance. Nurses' application of the South African Triage Scale is prone to errors, ${ }^{17}$ and utilisation of the score as a means of confirming onscene discharge may therefore have been underestimated. Other limitations include the use of discharge from the ED as a comparator to different practices regarding decision-making in the nontransportation of patients in other countries. No patients were followed-up with to assess changes that may have impacted their discharge decision. Patient demographics were also not collected, and previous studies have shown that older patients are more likely to be under-triaged by paramedics and sustain more harm from pre-hospital discharge than younger patients. ${ }^{8,16}$

\section{CONCLUSION}

Pre-hospital on-scene discharge by ECPs may be a mechanism to alleviate hospital overcrowding in a failing public healthcare system. Methods to perform this safely and effectively need to be in place prior to implementation in order to prevent deleterious effects from miss-assessed patients. Comparing the potential on-scene discharge patient with the patient admitted or discharged from the ED, however, may not ideally determine the appropriateness thereof.

\section{CONFLICTS OF INTEREST}

The authors report no conflicts of interest. 


\section{AUTHOR CONTRIBUTIONS}

CV-L conceptualised the study. SS collected the data. All authors were involved in data analysis and interpretation of data. LG drafted the initial version of the manuscript. All authors revised, edited and substantially contributed to the critical revision of the manuscript. Final approval of the version to be published is made by all authors who agree to be accountable for all aspects of the work in ensuring that questions related to the accuracy or integrity of any part of the work are appropriately investigated and resolved. LG takes responsibility for the paper as a whole.

\section{REFERENCES}

1. Mayosi BM, Benatar SR. Health and health care in South Africa-20 years after Mandela. N Engl J Med. 2014;371:1344-53.

2. Strachan B, Zabow T, Spuy Z van der. More doctors and dentists are needed in South Africa. S Afr Med J. 2011;101:523-8.

3. Coovadia H, Jewkes R, Barron P, D DS, McIntyre D. The health and health system of South Africa: Historical roots of current public health challenges. Lancet 2009;374:817-34.

4. Henderson K, Boyle A. Exit block in the emergency department: Recognition and consequences. Br J Hosp Med (Lond). 2014;75:6236.

5. Peyravi M, Örtenwall P, Khorram-Manesh A. Can medical decision-making at the scene by staff reduce the number of unnecessary ambulance transportations, but still be safe? PLOS Curr. 2015;30:ecurrents.dis.f426e7108516af698c8debf18810aa0a.

6. Gratton MC, Ellison SR, J JH, Ma OJ. Prospective determination of medical necessity for ambulance transport by paramedics. Prehosp Emerg Care. 2003;7:466-9.

7. Guidelines for good practice in the health care professions. booklet 3: national patients' rights charter. Pretoria: Health Professions Council of South Africa, 2016. (https : / / www . hpcsa . co . za / Uploads / Professional _ Practice / Ethics Booklet.pdf) (visited on 01/15/2019).

8. Ebben RHA, Vloet LCM, Speijers RF, et al. A patient-safety and professional perspective on non-conveyance in ambulance care: A systematic review. Scand J Trauma Resusc Emerg Med. 2017;25:71.
9. Tohira H, Fatovich D, Williams T, et al. Which patients should be transported to the emergency department? A perpetual prehospital dilemma. Emerg Med Austalas. 2016;28:64753.

10. Breeman W, Poublon WNA, Verhofstad MHJ, Lieshout EMMV. Safety of onscene medical care by ems nurses in nontransported patients: A prospective, observational study. Scand J Trauma Resusc Emerg Med. 2018;26:79.

11. Clinical practice guidelines for emergency care providers. Pretoria: Health Professions Council of South Africa, 2018. (https : / / www . hpcsa - co - za / Uploads / EMB / CLINICAL $\% 20$ PRACTICE 20 GUIDELINES $\%$ $20 \div 20$ - \%2OPROTOCOLS - 2OJULY 202018 .pdf) (visited on 01/15/2019).

12. The higher education qualifications subframework. Pretoria: Council on Higher Education, 2013. (https : / / www . che.ac. za/ sites/default/files/publications/ $\mathrm{CHE} \div 20$ - 20Higher 20 Education 20Qualification $\%$ OFramework $\% 20$ 20EDITED 20171013 . pdf) (visited on 02/24/2019).

13. Vincent-Lambert C. A framework for articulation between the emergency care technician certificate and the emergency medical care professional degree. PhD thesis. Unioversity of the Free State, 2012.

14. Geldenhuys N. The use of ambulances to transport patients to the Emergency Department of a public hospital in Johannesburg. Research rep. University of Johannesburg, 2011.

15. Helen Joseph Hospital. Johannesburg: Gauteng Provincial Government, 2018. (http : / / wWw . gauteng . gov . za/government / departments / health / services / hospitals / Pages / Helen - Joseph Hospital .aspx) (visited on 01/15/2019).

16. Tohira H, Fatovich D, Williams $\mathrm{T}$, et al. Is it appropriate for patients to be discharged at the scene by paramedics? Prehosp Emerg Care. 2016;20:539-49.

17. Goldstein LN, Morrow LM, Sallie TA, et al. The accuracy of nurse performance of the triage process in a tertiary hospital Emergency Department in Gauteng Province, South Africa. S Afr Med. J. 2017;107:243-7. 